\title{
Atividades físicas no contexto urbano saudável: a experiência de Conchal [SP]
}

Dossier de Pesquisa : CidAde SAUdável

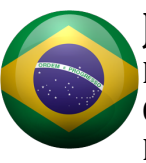
Jussara Conceição Guarnieri

Mestre em Arquitetura, Tecnologia e Cidade pela Universidade Estadual de Campinas, Fisioterapeuta pelo Centro Universitário Hermínio Ometto de Araras, graduada em Educação Física pela Universidade Estadual Paulista Júlio de Mesquita Filho. Conchal [SP] Brasil. <jussaraguarnieri@hotmail.com>.

\section{Resumo}

Na Carta Magna do Brasil, a Constituição Brasileira (1988) traz premissas para o desenvolvimento urbano através da função social da cidade proporcionado assim, uma vida saudável e digna de ser vivida. Relatar a implantação de um Projeto de Atividades Físicas em um município de pequeno porte pertencente a Rede de Municípios Potencialmente Saudáveis (RMPS para promover o urbano saudável. A metodologia é descritiva, sendo um estudo de caso a partir de iniciativas da Política Nacional da Promoção da Saúde (PNaPS) do Brasil de 2014 com a coleta dos resultados nos anos de 2010 a 2018 no Departamento Municipal de Saúde do município de Conchal [SP, Brasil]. A implantação de projetos de Atividades Físicas foi realizada através da criação de um Comitê Local, baseado na metodologia da RMPS, que proporcionou o mapeamento das ações desenvolvidas no território desse município e a gestão saudável do repasse federal do Ministério da Saúde (MS) desde 2010 destinado para implantação e implementação de projetos de promoção da saúde no combate ao sedentarismo. 0 projeto proporcionou o envolvimento, participação e comprometimento dos gestores locais e o empoderamento da comunidade, através das políticas de Promoção da Saúde vigentes com enfoque no urbanismo saudável. A participação do município na RMPS contribui no que diz respeito à promoção de saúde e trocas de experiências com outros municípios.

\section{Palavras-chave}

Atividades Físicas. Planejamento Urbano. Cidade Saudável.

\section{Physical activities in the healthy urban context: the Conchal city [estate of Sao Paulo, Brazil] experience}

\begin{abstract}
In the Magna Carta of Brazil, the Brazilian Constitution (1988) brings us premises for urban development through the social function of the city, thus providing a healthy life worth living. This study aims to report on the implantation of a Physical Activities Project in a small municipality belonging to the Network of Potentially Healthy Municipalities (RMPS), whose focus is to promote a healthy urban environment. The methodology of this study is descriptive from the National Policy for Health Promotion (PNaPS) in 2014 in Brazil and was a case study with the collection of results in the years 2010 to 2018 in the Municipal Department of Health of the city of Conchal [estate of Sao Paulo, Brazil]. The implantation of Physical Activity projects was accomplished through the creation of a Local Committee, based on the methodology of the RMPS, which provided a mapping of the actions developed in the territory of that municipality and the healthy management of the federal pass-through of the Ministry of Health (MS) since 2010 destined for implantation and implementation of health promotion projects in the fight against sedentarism. It is important that the municipal representatives comprehend the current policies of the health promotion with a focus on urbanism. The participation of the municipality in the RMPS contributes with regard to the promotion of health and exchanges of experiences with other municipalities.
\end{abstract}

\section{Keywords}

Physical activities. Urban Planning. Health city. 


\section{Introdução}

As premissas do planejamento urbano associadas com as da promoção da saúde pode proporcionar uma cidade saudável na medida que programas de qualidade de vida são consequências as ações integradas dos dois campos.

O planejamento da cidade, com enfoque na qualidade de vida e no bem-estar dos munícipes deve viabilizar espaços que permitam aos seus moradores praticarem por exemplo atividades físicas prevista na Lei 8080, que dispõe sobre as condições da promoção, proteção e recuperação da saúde e a organização dos serviços e especificamente, no artigo 3 aponta que são os níveis de saúde que expressam a organização social e econômica do País e ainda cita que a atividade física é um determinante e condicionante da saúde (Brasil, 1990). O Estatuto da Cidades descreve, da mesma forma, a importância do lazer como diretriz para o direito e desenvolvimento das cidades sustentáveis (Brasil, 2001).

Para a Organização Mundial da Saúde (OMS, ou "WHO" em inglês), a saúde global está sendo influenciada pela rápida urbanização não planejada e pelo acelerado processo de globalização, os quais resultam em ambientes e comportamentos não saudáveis além do surgimento de fatores de risco para doenças crônicas não transmissíveis (WHO, 2010).

A atividade física é definida como qualquer movimento corporal produzido pelos músculos esqueléticos que requer gasto de energia, inclui o exercício, bem como outras atividades que envolve movimento corporal e são feitas como parte de jogar, trabalhar, transporte ativo, tarefas domésticas e atividades recreativas (WHO, 2011).

Reforçando a importância, Franklin (2004) descreve que a história da atividade física acompanha a atividade laboral, onde séculos atrás a população que vivia em fazendas e trabalhavam no campo tinham intensas atividades físicas no desenvolver do seu trabalho diário e com o êxodo do campo para as áreas urbanas esse cenário mudou. 0 mesmo autor refere que apenas cerca de $5 \%$ da população mundial desenvolve atividade laboral por meio da atividade agrícola. Segundo o mesmo autor, o período econômico pós-industrial é caracterizado pela "mesa e o terminal do computador" provocando uma redução de movimentos corporais voltadas para o trabalho. Com a invenção das novas máquinas, como por exemplo, máquina de lavar roupas, lavar louças e de cortar grama, algumas atividades domésticas também reduziram a possibilidade de exercitar o corpo em função do trabalho doméstico. E como desenvolvimento econômico acentuou-se a preocupação com a produção de automóveis e seu designer reduzindo a preocupação com a criação de espaços urbanos para a locomoção, caminhando ou usando uma bicicleta (Franklin, 2004).

Segundo a Organização Mundial de Saúde (OMS, 2010) a inatividade física é responsável como o fator de risco para a quarta principal razão da a mortalidade mundial $6 \%$ das mortes em todo o mundo). Isto segue a pressão arterial elevada, com 13\%, o tabaco com $9 \%$ e de glicemia elevada com $6 \%$ (diabetes). 0 excesso de peso e a obesidade são responsáveis por $5 \%$ da mortalidade no mundo.

Além do aumento dos fatores de risco, a obesidade, segundo Malta (2009), como pressão arterial elevada, alta taxa de açúcar no sangue e excesso de peso, a inatividade física é estimada como sendo o principal motivo com aproximadamente $21-25 \%$ câncer de mama e de câncer de cólon, $27 \%$ de diabetes e aproximadamente $30 \%$ da carga isquêmica cardíaca, além disso, doenças não transmissíveis respondem hoje por quase metade do total mundial das doenças.

É evidente que o estilo de vida mudou provocando o sedentarismo e como consequências a obesidade, diabetes, hipertensão, depressão, entre outras. Os níveis de inatividade física estão aumentando em muitos países com maiores implicações para a saúde geral das pessoas e para prevalência de Doenças Crônicas Não Transmissíveis (DCNT's), como doenças cardiovasculares, diabetes e câncer (WHO, 2010).

Para Malta et al., 2009 a mudança do perfil epidemiológico no país, com predominância das doenças não transmissíveis é uma consequência da urbanização, da mudança nos estilos de vida 
e da globalização e trata-se de um mal que pode ser prevenido, geralmente a um custo menor do que o das intervenções curativo-assistenciais, reforçando assim, a prática regular de atividade física apresenta uma relação inversa com risco com as DCNT's e tem um efeito positivo na qualidade de vida das pessoas, com ênfase à redução do sedentarismo, e melhoria da saúde individual e coletiva (Oehlschlaeger, 2004).

A saúde global está sendo influenciado por três vertentes: o envelhecimento da população, rápida urbanização não planejada e a globalização. 0 crescimento da prevalência de DCNT's e seus fatores de risco tornaram-se um problema mundial que afeta tanto aos países de baixa quanto a de média renda e quase $45 \%$ das doenças em adultos nesses países são atribuídas as DCNT's e muitos países de baixa e média renda estão começando a sofrer o duplo fardo tanto das doenças crônicas transmissíveis bem como as não transmissíveis e os sistemas de saúde desses países estão tendo que lidar com os custos adicionais de tratamento de ambos (OMS, 2010).

Estudos demonstram que a participação em atividade física regular reduz o risco de doença coronariana e acidente vascular cerebral, diabetes, hipertensão, câncer de cólon, câncer de mama e depressão (Matsudo, 2010). Além disso, a atividade física é um fator determinante do gasto energético e, portanto, é fundamental para balanço de ingestão de calorias e de controle de peso.

O presente trabalho tem como objetivo relatar a implantação de Projetos de Atividades Físicas em um município de pequeno porte brasileiro pertencente à Rede de Municípios Potencialmente Saudáveis (RMPS) em que o enfoque é promover o urbano saudável.

\section{Metodologia}

A metodologia deste estudo é descritiva a partir de iniciativas com a PNaPS e foi um estudo de caso com a coleta dos resultados entre os anos de 2010 a 2018 no Departamento Municipal de Saúde, do município de Conchal [SP]. Para tanto, foram consultados documentos, como os projetos que foram enviados ao Ministério da Saúde, portarias estabelecidas, site de Rede de Municípios Potencialmente Saudáveis, da Organização Mundial de Saúde, além da revisão de artigos científicos na base bibliográfica da Scielo e Lilacs e base de dados do Polo Academia da Saúde do município citado.

\subsection{Conchal [SP], cidade pertencente à Rede de Municípios Potencialmente Saudáveis}

Com uma população de aproximadamente 27.242 habitantes (IBGE, 2010), o município ora citado é, desde 2010, parte integrante da Rede de Municípios Potencialmente Saudáveis (RMPS) que contribui para implementação de políticas públicas saudáveis, tornando viável, expandindo e direcionando projetos e estratégias que visam melhorar o ambiente urbano de forma a torná-lo saudável para a população.

A RMPS (2003) tem por objetivo prioritário a viabilização, expansão e manutenção de projetos da Organização-Pan-americana da Saúde (OPAS) para as "Cidades Saudáveis". Estas pautas constituem-se em construir políticas públicas saudáveis, promover a participação social de todos os setores, implantar iniciativas sustentáveis, agregar a contribuição e atuação de múltiplos setores e parceiros.

Segundo a mesma rede, há a necessidade de instrumentar a administração municipal para que desenvolva políticas de governo único e integrado subsidiando gestores, técnicos, acadêmicos, organizações e sociedade para a construção de políticas públicas que introduzam a promoção da saúde e melhoria da qualidade de vida como prioridade no desenvolvimento de seus projetos. (Sperandio, 2003).

A melhor forma de se dar início a um trabalho em rede é permitir que os participantes aprendam uns com os outros, de forma simultânea e progressiva, mediante a contribuição e atuação em assuntos, ideias, experiências, relatos de problemas e respectivas soluções, apresentação de resultados de pesquisa e qualquer outra forma de contribuição pertinente e de interesse mútuo. (OPAS/OMS, 2010). 
A maioria das redes é formada sem pretensão alguma de organização, e algumas pessoas que possuem interesse em comum se reúnem e dão sequência espontânea a essas conversas e ações, agindo, despretensiosamente e sem regras, como se tudo fosse apenas uma grande "rede social". A certa altura percebe, mediante a expansão dos participantes e colaboradores que aumenta consideravelmente, a necessidade da formalização, do estabelecimento de norteadores, de definição de regras e organização. São essas redes que colaboram muito com a formação das cidades saudáveis, definidas pela Organização Mundial da Saúde (OMS, 2010).

O município é considerado "cidade saudável" quando conseguem impactar a sociedade, suas organizações que a representam, bem como as autoridades políticas em exercício e desenvolvem ações com comprometimento e empenho visando a promoção da saúde e a melhoria da qualidade de vida de seus moradores e residentes, com a participação social.

\subsection{Política Nacional de Promoção da Saúde como promotora da cidade saudável}

Com a aprovação da Política Nacional de Promoção da Saúde (PNaPS) em 2006 no Brasil e revisada em 2014 ratificou a institucionalização da promoção da saúde no Sistema Único de Saúde (SUS), em atenção a ampliação do conceito de saúde e valorização da abordagem epidemiológica foram eleitas áreas temáticas prioritárias para a implementação da PNaPS do Brasil, destacandose a atividades físicas corporais como fator de proteção da saúde (Malta et al., 2009).

Destaca-se a relevância da intersetorialidade, visto que, a opção de um indivíduo por uma vida ativa e saudável depende da oferta, da oportunidade de acesso a espaços favoráveis a prática do lazer e/ou exercício físico, como pistas de caminhadas, ciclovias, praças públicas, espaços para a prática de esporte, além da garantia da segurança, infraestrutura adequada, áreas arborizadas, acesso pelo transporte público, entre outros direitos de cidadania.

A questão de praticar ou não a atividade física ligada remete ao planejamento urbano e a mobilidade urbana, pois deve considerar os modos como indivíduos e coletividades transitam, ocupam e criam identidade territorial e utilitarista com os espaços públicos de lazer. E para a promoção da saúde é fundamental estabelecer parcerias com todos os setores da administração pública, empresas, organizações não governamentais (ONG), para induzir mudanças sociais, econômicas e ambientais que favoreçam políticas públicas vinculadas a garantia dos direitos da cidadania e autonomia dos sujeitos e coletividades.

Segundo Brandão (2010), para que os municípios brasileiros adotem programas de promoção da saúde é necessário um comprometimento da parte da gestão pública empoderando a comunidade, fortalecendo a participação social na construção de projetos que beneficiam a população local através de políticas públicas saudáveis. E Akerman et al. (2002), reforça que o governo local deve desenvolver diálogos para que as condições de saúde e dos determinantes sociais possam estar em sintonia com o plano diretor dos municípios através do planejamento urbano para a construção de cidades saudáveis propiciando uma melhor qualidade de vida aos seus munícipes.

O Plano Diretor, é apontado segundo Francisco Filho (2010), como um o mecanismo essencial de políticas urbanas para que a cidade se desenvolva transformando lugares, englobando territórios, que os munícipes se sentem pertencentes ao local calcados nas premissas dos documentos norteadores do planejamento urbano.

\section{Resultados e discussões}

A implantação dos programas de Atividades Físicas foi realizada através da criação do Comitê Local, baseado na metodologia da RMPS, que proporcionou o mapeamento das ações desenvolvidas no território de Conchal [SP] e a gestão saudável do recurso do Ministério da Saúde destinado para implantação de projetos de promoção da saúde no combate ao sedentarismo e consequentemente as DCNT`s. Durante as reuniões intersetoriais os itens monitorados foram os documentos oficiais, filmagens, relatórios das aulas, registros fotográfico e roda de conversas entre os participantes. 
A rede foi formada por um Grupo de Trabalho Intersetorial que se reúnem e discutem projetos do município nos departamentos que visam melhorias na qualidade de vida da população. Tratase de um trabalho articulado entre as secretarias e departamentos que tem, entre seus projetos prioritários, o fortalecimento da rede local, promover a participação social nos projetos e acesso a informação.

Participam dessa rede, o Departamento de Saúde, Meio Ambiente, Obras, Rendas, Finanças, Recurso Humano, Segurança Pública, jurídico articulado pela Rede de Municípios Potencialmente Saudáveis (RMPS), que é vinculada a Faculdade de Engenharia, Arquitetura e Urbanismo da Universidade Estadual de Campinas (UNICAMP).

Para o desenvolvimento de Conchal na perspectiva de uma cidade saudável foram realizadas diferentes reuniões intersetorias e com representações da comunidade para as decisões coletivas em particular o tema atividades físicas na Cidade. Para tanto, foi pleiteado pelo município de Conchal [SP], em 2010 o repasse financeiro federal através da portaria de no 139 de 09.09 .09 do Ministério da Saúde para implantação do projeto contemplando dois dos sete eixos da Política Nacional da Promoção da Saúde (PNPS): atividades físicas/ práticas corporais e alimentação saudável nas Unidades de Saúde da Família (USF’s) do município.

As Unidades de Saúde da Família (USF's) passaram a ser implantadas no município de Conchal[SP] em 2001 e as mais recentes foram implantadas em 2006, outras receberam reformas e melhorias em 2018, totalizando 05 (cinco) unidades de Saúde da Família e 01 Unidade Básica Saúde (UBS). Com o financiamento do Ministério da Saúde foi organizado mini academias que pudessem desenvolver atividades físicas como: alongamentos, ginástica localizada, pilates com Bola, jogos recreativos e atividades lúdicas. Esse projeto objetivou realizar um trabalho multidisciplinar e de atenção integral a saúde dos hipertensos e diabéticos visando reduzir as consequências destas patologias e a promoção de saúde, ampliando as ações na prevenção de doenças através da prática de atividade física e corporal nos usuários das Unidades de Saúde da Família, proporcionando atividades educativas em saúde, abrangendo: alimentação saudável, incentivo da prática de atividade física e atividades culturais.

Essas ações propiciaram benefícios aos usuários como a mudança de estilo de vida, aquisição de hábitos saudáveis e a prática de atividade física que são fundamentais para o bem-estar e a qualidade de vida da população.

Para tanto houve a necessidade de espaços físicos e profissionais capacitados para desenvolverem as atividades. 0 trabalho em rede desenvolvido no município contribuiu para a mobilização da sociedade civil na busca de soluções alternativas para garantir os resultados do projeto, tais como: uso de espaços da própria comunidade como salão paroquial, garagem, locais públicos, etc. Os profissionais necessários foram remanejados de outros setores públicos como Departamento de Esportes.

Em continuidade às ações de promoção da saúde que estavam ocorrendo, o município pleiteou através da portaria no 184 de 24.06.10, mais um projeto de "Promoção da Saúde com Foco na Redução do Uso de Álcool e Drogas e Fomentação do Desenvolvimento Sustentável" abrangendo três dos sete eixos da Política Nacional da Promoção da Saúde (PNaPS) durante o ano de 2011, que são: Alimentação Saudável, Redução do uso abusivo de Álcool e Drogas e Desenvolvimento Sustentável.

Foi por meio de repasse federal que as ações e programas para Ambientes Livres do Tabaco (ALT), além do tratamento para parar de fumar e desenvolvimento de grupos anti-tabaco "Corta Essa" foram desenvolvidos no Centro de Especialidades Médicas de Conchal (CEMEC), nas Unidades de Saúde da Família e nos espaços públicos. Outros projetos como a Horta Comunitária e Família sem Drogas também são beneficiados pelo mesmo repasse.

Como resultado dos trabalhos desenvolvidos no município de Conchal [SP] foi possibilitado a participação como membro do Projeto Agita São Paulo, projeto este que foi criado pelo Centro de Estudos do Laboratório de Aptidão Física de São Caetano do Sul - CELAFISCS em parceria com a 
Secretaria de Saúde do Estado de São Paulo. O projeto nasceu com a ideia de aumentar o nível de conhecimento da população sobre a importância da atividade física e também para incentivar a prática de atividade física diária como forma de combater diversas doenças, como o sedentarismo. Este projeto enfatiza principalmente a troca de práticas pouco ativas do dia-a-dia por exercícios que gastem mais energia, como levar o cachorro para passear, não andar excessivamente de carro, andar de bicicleta, subir e descer escadas e dicas de alongamento antes de caminhar.

Além desse programa, com a portaria 1.707 de 23 de setembro de 2016 (Brasil, 2016), atuando com a promoção da saúde e na prevenção de doenças, o Ministério da Saúde redefine as regras e os critérios referentes aos investimentos para os Polos Academia da Saúde no município para que o programa seja mais um espaço urbano para práticas de atividades físicas orientada pelo profissional de educação física da saúde.

O programa Academia da Saúde no município de Conchal está instituído desde agosto/2014 no município de Conchal, onde mais de 4.000 usuários do Sistema Único de Saúde já passaram pelo projeto, praticando alguma atividade física para combate ao sedentarismo, consequentemente diminuindo as Doenças Crônicas Não Transmissíveis tais como: diabetes, obesidade, Hipertensão Arterial, entre outros. 0 fato demonstra a importância de ações transdisciplinar para atendimento as áreas que existem interfaces e integram para os desejos e bem comum.

\section{Considerações finais}

Entende-se a importância da compreensão pelos representantes dos municípios brasileiros das políticas de Promoção da Saúde vigentes com enfoque no urbanismo e concluiu-se ser necessário melhorias nos espaços urbanos criando-se mecanismo e diferentes estratégias de atuação dos gestores para a comunidade.

0 trabalho intersetorial, com participação da comunidade alcançam resultados e podem colaborar com a longevidade de populações com qualidade. 0 desenvolvimento de uma cidade saudável necessita de um planejamento urbano, onde a função social da cidade e a participação social com a autonomia das pessoas sejam respeitadas e identificadas e assim, que os desejos sejam colocados como prioridade na agenda. E com essa autonomia dos munícipes, os projetos de promoção da saúde ganham sustentabilidade e continuidade independente de mudanças políticas.

A participação do município na Rede de Municípios Potencialmente Saudáveis (RMPS) em algumas cidades brasileiras contribui no que diz respeito à promoção de saúde e trocas de experiências com outros municípios, bem como o envolvimento da sociedade civil na busca de melhorias do seu bem viver na perspectiva do urbano saudável.

\section{Referências}

Akerman et al. (2002). Avaliação em promoção da saúde: foco no município saudável. Revista Saúde Pública, 36(5): 638-46, Disponível em: <https://www.scielosp.org/scielo.php? script=sci_arttext\&pid=S0034-89102002000600016>. Acesso em 25 nov. 2018.

Brasil (1988). Constituição da República Federativa do Brasil. Brasília, DF, Senado.

Brasil (2001). Lei 10.257 de 10 de julho de 2001, o "Estatuto das Cidades", regulamenta o capítulo "Política Urbana" da Constituição brasileira.

Brasil, Ministério da Saúde. Secretaria de Vigilância em Saúde (2006). Política Nacional de Promoção da Saúde. Brasília, DF. 60p. (série B. Textos Básicos de Saúde) (Série Pactos pela Saúde , 7).

Brasil (2016). Portaria 1.707 de 23 de setembro de 2016. Recuperado de: <http://www.brasilsus.com.br/index.php/legislacoes/gabinete-do-ministro/9218-portaria-n-1-707de-23-de-setembro-de-2016>.

(C) Labor \& Engenho, Campinas [SP] Brasil, v.12, n.4, p.533-539, out./dez. 2018. 
Brandão, I. R. (2010). Na trilha do município saudável. In: Organização Pan-Americana da Saúde, A. Sperandio et al. (org.). Políticas Integradas em Rede e Construção de Espaços Saudáveis: boas práticas para a iniciativa dos rostos, vozes e lugares (p.37). Brasília: OPAS.

Franscisco Filho, L. L. (2010). In: Políticas Integradas em Rede e Construção de Espaços Saudáveis: boas práticas para a iniciativa dos rostos, vozes e lugares (p.159). Brasília: OPAS.

Franklin, T. et al. (2004). Walkable streets. New Urban Futures, 10: 5-7. July.

IBGE. Instituto Brasileiro de Geografia e Estatística (2010). Contagem Populacional.

Malta, D. C. et al. (2009). A Política Nacional de Promoção da Saúde e a agenda da atividade física no contexto do SUS. Epidemiol. Serv. Saúde, Brasília, 18, pp. 79-86, jan./mar.

Malta, D. C. et al. (2009) Padrão de atividade física em adultos brasileiros: resultados de um inquérito por entrevistas telefônicas. Epidemiol. Serv. Saúde, Brasília, 18, pp. 7-16, jan./mar.

Oehlschlaeger, M. H., Pinheiro, R. T., Horta, B., Gelatti, C., \& Santana, P. (2004). Prevalência e fatores associados ao sedentarismo em adolescentes de área urbana. Revista Saúde Pública, 38, pp. 157-163.

OPAS. Organização Pan-Americana de Saúde (2008). Gestão em rede na OPAS/OMS Brasil. Conceitos, práticas e lições aprendidas. Brasília: OPAS.

RMPS. Rede de Municípios Potencialmente Saudáveis (s./d.). Disponível em: <www.redemunicipiosps.org.br>. Acesso em 25 nov. 2018.

Sperandio, A. M. G., \& Malo, M. (2006). Relembrando os princípios da Rede de Municípios Potencialmente Saudáveis. O plano diretor: uma ferramenta para o desenvolvimento das políticas públicas de um município potencialmente saudável. Campinas: Universidade de Campinas, Departamento de Medicina Preventiva; \& Organização Pan-Americana da Saúde.

Sperandio A. M. G. (2010). A promoção da saúde construída em Rede. In: A.M.G. Sperandio, D. G. Marchín, M. A. B. Fortunato (orgs.). Políticas Integradas em Rede e a Construção de Espaços Saudáveis: boas práticas para a Iniciativa do Rostos, Vozes e Lugares. Organização Pan-Americana da Saúde. Org. Sperandio, A.M.G.; Marchín D.G; Fortunato M.A.B. Brasília: Organização Pan-Americana da Saúde.

Sperandio A. M. G., Veríssimo, L. C. G., \& Palácios A. L. O. (2011). O processo de desenvolvimento de políticas públicas saudáveis integradas em rede: a experiência de Maringá - PR. Ano VII [№ 015] abr./jun. pp. 10-28.

WHO. World Health Organization (2010). Global recommendations on physical activity for health. Disponível em: <http://www.who.int/dietphysicalactivity/factsheet_recommendations/en/>. Acesso em 12 mar. 2017. 